\title{
Bodegas subterráneas excavadas en tierra: Características de los suelos en la Ribera del Duero (España)
}

\author{
Wine cellars built underground: soil properties in the \\ "Ribera del Duero" (Spain) \\ I. Cañas ${ }^{(*)}$, J. Cid-Falceto ${ }^{(*)}, \underline{\text { F. R. Mazarrón }}{ }^{(*)}$
}

RESUMEN

Las propiedades de la tierra influyen de manera decisiva en las condiciones interiores, la integridad y la durabilidad de las bodegas subterráneas. El principal objetivo del presente artículo es determinar las características de los suelos donde se excavan las bodegas subterráneas. Los resultados demuestran que la mayoría de los suelos presentan unas características de plasticidad y expansibilidad muy similares. Se concentran en una región muy concreta de la carta de plasticidad o presentan plasticidad nula. Los suelos con alta plasticidad no son habituales, debido al mayor riesgo de sufrir expansiones $y$ provocar problemas en las bodegas. En cuanto a granulometría predominan los limos y arenas menores de 0,4 mm, con un contenido en arcillas menor. No se han encontrado bodegas excavadas en terrenos con muy alta plasticidad, ni tampoco donde predominen gravas o arenas limpias. Recomendamos que las nuevas bodegas sean excavadas en terrenos de similares características.

$160-17$

Palabras clave: Propiedades del suelo; construcción subterránea; bodegas; construcción bioclimática

\section{SUMMARY}

The ground properties have a decisive effect on the interior conditions, as well as the integrity and durability of the underground wine cellars. The main objective of this paper is to determine the characteristics of the soils where these underground wine cellars have been dug. The results show that most of the soils present very similar plasticity and swelling properties. They are either concentrated in a very specific region of the plasticity chart or present zero plasticity. High-plasticity soils are uncommon, owing to the higher risk of swelling leading to stability problems in the wine cellars. In terms of grain size, silts and sands under $0.4 \mathrm{~mm}$ predominate, with a somewhat lower presence of clays. No wine cellars were found that had been dug in earth with very high plasticity or where gravels or clean sands predominate. We recommend that any new wine cellars should be excavated in earth with similar characteristics.
Keywords: Ground properties; underground building; wine cellar; bioclimatic construction.

\footnotetext{
(*) ETSIA-Universidad Politécnica de Madrid (España). 
1. Localización de la zona de estudio: Ribera del Duero.

2. Representación de una bodega subterránea típica

\section{INTRODUCCIÓN}

La edificación en tierra aporta criterios de sostenibilidad en la edificación (1), además de utilizarse cada vez más como material de construcción (2). Entre las técnicas de construcción con tierra destacan las construcciones subterráneas. El hombre ha excavado durante siglos miles de bodegas subterráneas, en las cuales ha criado y envejecido el vino. La inercia térmica y las propiedades de la tierra proporcionan estabilidad térmica y las condiciones necesarias para la conservación del vino con un coste energético nulo (3). Las condiciones higrotérmicas de las bodegas subterráneas han sido ampliamente estudiadas (4) (5) (6) (7), demostrando que las propiedades de la tierra son uno de los factores que más influye en el ambiente interior.

Por lo general, estas bodegas se caracterizan por estar excavadas en terrenos fácilmente perforables con herramientas sencillas. En la mayoría de los casos no presentan estructuras portantes ni de refuerzo interior, salvo en construcciones de gran anchura de la cueva. Se construían gracias al conocimiento transmitido durante siglos de generación en generación, adquirido mediante ensayo y error. Sin embargo, ese conocimiento se perdió hace tiempo, y actualmente se desconocen los criterios para seleccionar la ubicación más adecuada y el tipo de terreno.

La elección de un suelo con propiedades inadecuadas podría ocasionar problemas que afecten a la integridad de la construcción o a las condiciones interiores, como sucede en otras tipologías constructivas. Así, son numerosos los estudios que tratan los problemas de compresibilidad, expansibilidad e inadecuación de los suelos para la construcción de túneles (8) (9) (10) (11).

Ante la creciente demanda por parte de bodegas de prestigio de recrear las condiciones higrotérmicas de las antiguas bodegas tradicionales para la producción de vinos de calidad, pretendemos determinar las características de terrenos adecuados para la construcción de nuevas bodegas subterráneas. Para ello se han estudiado las propiedades de la tierra en la que están excavadas bodegas centenarias que se han conservado hasta nuestros días. La zona de estudio seleccionada es una de las regiones con mayor tradición vitivinícola y mayor número de bodegas subterráneas de España: La Ribera del Duero.

En el sector vitivinícola, las bodegas subterráneas tradicionales pueden ser una buena solución bioclimática al problema energético actual.

\section{BODEGAS DE ESTUDIO}

\subsection{Zona}

El estudio se centra en la región que delimita la Denominación de Origen Ribera del Duero (Figura 1). Las tierras de esta Denominación se sitúan en la meseta norte de España. El río Duero es el eje que une 117 núcleos rurales, extendidos en una franja de unos $115 \mathrm{~km}$ de longitud y 35 de anchura. El relieve de la zona oscila entre lomas interfluviales, con cotas de más de 900 metros, y valles entre los 750 y 850 metros.

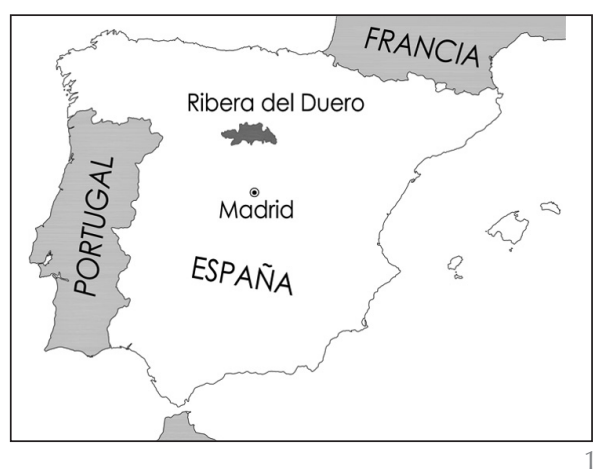

Presenta un clima mediterráneo continentalizado, caracterizado por unos inviernos largos y fríos, con veranos cortos no muy calurosos y secos. La precipitación media anual está entre 400 y 600 mm, y se concentra principalmente en el otoño y la primavera. La temperatura media anual suele estar próxima a los $11^{\circ} \mathrm{C}$, oscilando entre los $20^{\circ} \mathrm{C}$ de media mensual en julio y los $3^{\circ} \mathrm{C}$ de enero.

\subsection{Características y técnica constructiva}

La Figura 2 muestra las partes principales de una bodega típica de la región. Una descripción más detallada puede encontrarse en Pardo y Guerrero (12).

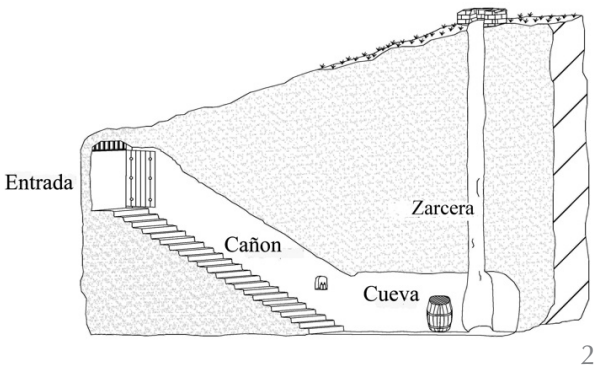

El proceso de construcción de una bodega subterránea tradicional se detalla a continuación (Figura 3):

1. En primer lugar se seleccionaba la ubicación en función de parámetros como la topografía, las características del suelo, la distancia al núcleo urbano, etc. Los terrenos con una ligera inclinación son los más adecuados para la construcción de las bodegas. 
2. La excavación se empezaba realizando un corte en la ladera, lo que se convertiría en la futura entrada de la bodega.

3. Se continuaba con la excavación del cañón o túnel de acceso a la cueva de la bodega, reforzándose la parte superior del cañón en el interior con mampostería. El material extraído se situaba por encima del cañón creando una loma, evitando el trabajo de transporte a sitios más alejados y consiguiendo una mayor masa térmica. Al mismo tiempo se iba excavando la chimenea de ventilación mediante la utilización de poleas para sacar el material extraído.

4. Una vez terminados el túnel y la chimenea se excavaba la cueva, con una sección más ancha que el cañón de acceso, depositando los materiales encima de ésta y del cañón. Para finalizar se introducían los utensilios para el proceso de elaboración del vino.

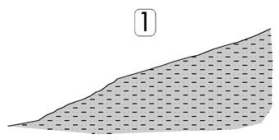

3

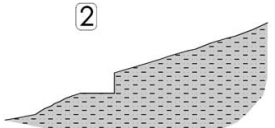

4

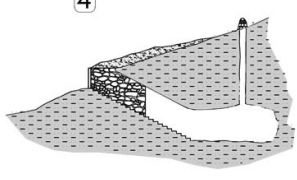

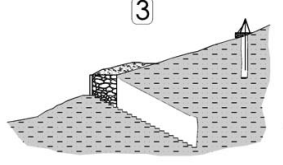

bera del Duero, identificando los barrios de bodegas existentes. Cada uno de estos núcleos fue catalogado, recogiendo la siguiente información: situación (coordenadas GPS); topografía; proximidad al núcleo urbano; número aproximado de bodegas; materiales de construcción empleados; orientación de los cañones; estado de conservación de las bodegas y fotografías.

La Figura 5 muestra la localización de los 106 pueblos donde se han identificado y catalogado barrios de bodegas, numerando aquellos en que se han tomado muestras para su análisis posterior.

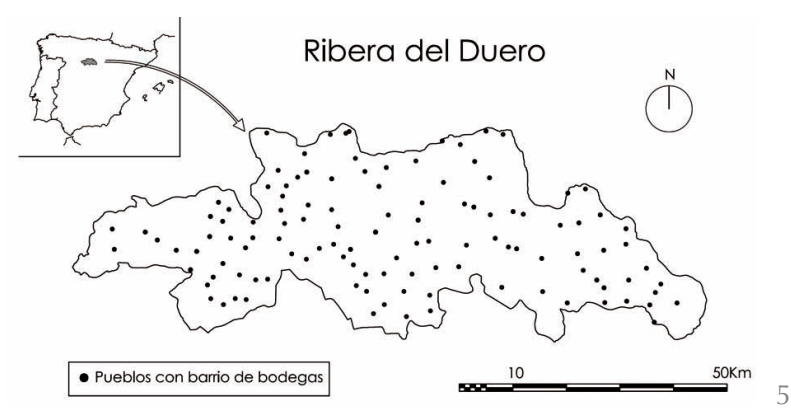

\subsection{Selección de las muestras}

Para el estudio de los terrenos se seleccionaron 31 muestras, lo que supone un $30 \%$ de los barrios de bodegas existentes (Figura 6). La selección se realizó proporcionalmente al número de barrios de bodegas existentes en cada una de las provincias por las que discurre la Ribera del Duero (Soria, BurgosSegovia y Valladolid), agrupando Burgos y Segovia en un mismo grupo al disponer esta última de una superficie muy pequeña en la Ribera (Figura 5). En una primera fase se seleccionaron aproximadamente el $20 \%$ de los barrios de bodegas existentes en cada una de las tres regiones, con muestras distribuidas desde las orillas del Duero hasta los lugares más alejados. Este proceso de selección estuvo fuertemente condicionado por factores como la facilidad para acceder a los barrios de bodegas, la disponibilidad de permisos para la toma de muestras, etc. Por ello, en una segunda fase, al disponer de mayor colaboración por parte de autoridades y propietarios en la zona de Soria, se decidió aumentar hasta un $50 \%$ de los barrios existentes en esta provincia, disponiendo así de una zona caracterizada con mayor detalle. En cada una de las localizaciones se tomaron muestras de una bodega representativa del conjunto.

Debido a que las bodegas son propiedad privada no fue posible conseguir el permiso de todos los propietarios para tomar las muestras del interior. Se permitió la toma de muestras interiores en 13 bodegas. No obstante teniendo en cuenta el sistema constructivo de las bodegas, en el que la
3. Proceso de construcción de una bodega tradicional.

4. Barrio-tipo de bodegas subterráneas. de bodegas existentes en la Ribera del Duero.
5. Localización de los 106 barrios

En primer lugar se identificaron y clasificaen la zona de estudio. En segundo lugar se fijó la metodología de muestreo y ensayos de laboratorio para determinar el tipo de suelo. Finalmente se llevó a cabo un análisis de los resultados.

\subsection{Identificación y catalogación de los barrios de bodegas}

Durante el año 2006 se visitaron los 117 núcleos urbanos que integran la D.O. Ri- 
6. Localización de los barrios de bodegas seleccionados. tierra extraída se amontonaba encima del cañón de acceso (Figura 3), es posible tener una muestra del suelo donde se realizaba la bodega tomándola en el exterior sin afectar a las paredes interiores.

Dado el gran volumen de tierra extraído de una bodega, y la posible existencia de estratos de composición no perfectamente homogénea, es lógico que la granulometría de las muestras interiores y exteriores no coincida exactamente. No obstante, este trabajo pretende caracterizar el terreno de las bodegas subterráneas que han aguantado el paso del tiempo, proporcionando un rango de valores orientativo. Por ello, pequeñas diferencias entre las muestras interiores y exteriores no son significativas para el objeto del estudio. Para tener un orden de magnitud de estas diferencias se tomaron muestras al azar del interior y exterior de las bodegas en seis localizaciones distintas, distribuidas proporcionalmente al número de barrios existentes en las tres regiones de estudio, confirmando con posterioridad que se trataba de suelos con granulometría y plasticidad similares.

De esta forma, se seleccionaron seis localizaciones con ejemplares de muestras interiores y exteriores, dieciocho con ejemplares de muestras exteriores y siete con ejemplares de muestras interiores, hasta completar las treinta y una muestras de las que disponemos (Figura 6).
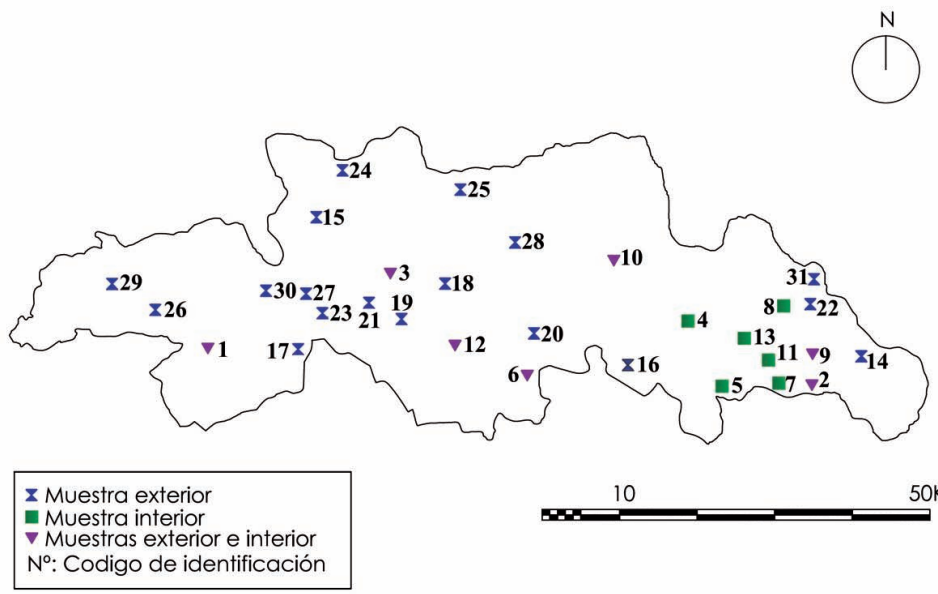

La metodología de toma de muestras aplicada fue similar para los dos tipos de muestras. En el caso de muestras exteriores, en primer lugar se eliminaba la capa de tierra vegetal (capa de 15 a $30 \mathrm{~cm}$ de la superficie del suelo) del terreno por encima del cañón de entrada mediante una pequeña azada. A continuación, se cogían unos 2-3 kg que se depositaban en una bolsa hermética. Con las muestras interiores se procedió con una metodología similar, cogiéndose las muestras de las paredes de la bodega tras haber eliminado la capa más superficial.

\subsection{Clasificación y caracterización de los suelos}

El principal objetivo de cualquier sistema de clasificación de suelos en el ámbito de la ingeniería es predecir las propiedades del suelo basadas en unas pocas pruebas, clasificando los suelos en grupos con similares características ingenieriles. Durante mucho tiempo se han utilizado principalmente dos clasificaciones geotécnicas para la descripción del suelo en aplicaciones de ingeniería civil: AASHTO y USCS (13).

El sistema USCS (Unified Soil Classification System) es la base para la identificación de suelos en una gran diversidad de propósitos de ingeniería civil. El USCS deriva de un sistema desarrollado por Casagrande para clasificación de suelos. El sistema divide a los suelos en tres grandes grupos:

- Suelos de granos gruesos, constituidos por gravas y arenas con menos del 50\% de finos que pasan por el tamiz $n^{\circ} 200$ ASTM. Los subgrupos se clasifican en función de la granulometría del suelo y de la plasticidad de la fracción que pasa por el tamiz $n^{\circ} 40$. Se utilizan los siguientes símbolos: G (grava) y $\mathrm{S}$ (arena), junto con un sufijo W (bien graduado) y $\mathrm{P}$ (mal graduado), o M (limo) y C (arcilla). Así por ejemplo, SM y SC corresponden a las gravas y arenas con una proporción importante de finos.

- Suelos de granos finos, arcillosos y limosos, constituidos por los suelos con 50\% o más de finos. Se utilizan los prefijos M (limo), C (arcilla) y O (materia orgánica), y los sufijos L (bajo LL) y H (alto LL). Para la clasificación de un suelo como arcilla o limo, se utiliza el gráfico de plasticidad. Por ejemplo, los suelos CL, ML son arcillas y limos de baja plasticidad, mientras que $\mathrm{CH}, \mathrm{MH}$ son de alta plasticidad.

- Suelos altamente orgánicos, constituidos fundamentalmente por materia orgánica. Se designan mediante el símbolo PT.

La clasificación adoptada por la American Associattion of State Highway Officials (AASHTO), es el sistema más utilizado en la clasificación de suelos en carreteras. Los suelos se clasifican en función de su comportamiento como capa de soporte o asiento del firme, en siete grupos (A-1, A-2, A-3, A-4, A-5, A-6 y A-7), según su granulometría (porcentaje que pasa por los tamices $n^{\circ} 200,40$ y 10) y plasticidad (Límites de Atterberg). Estos siete grupos se clasifican en dos grandes categorías, suelos granulares: A-1, A-2 y A-3 (con no más del $35 \%$ que pasa por el tamiz $\mathrm{n}^{\circ}$ 200) y suelos limo-arcillosos: A-4, A-5, A-6, A-7 (más del 35\% que pasa por el tamiz $n^{\circ}$ 200). Así por ejemplo, los suelos A-2-4 
son gravas y arenas limo-arcillosas, los A-4 son suelos limosos no plásticos o moderadamente plásticos, los A-6 suelos arcillosos plásticos y los A-7-5 y A-7-6 son suelos con un IP medio o elevado y que están sujetos a importantes cambios de volumen.

Numerosos estudios utilizan las clasificaciones mencionadas para la caracterización de los suelos y la determinación de sus características ingenieriles. Así por ejemplo, Al-rawas y Qamaruddin (14) se basan en los datos obtenidos de la carta de plasticidad y la clasificación USCS para determinar la expansibilidad y estudiar los problemas constructivos de suelos expansibles. Otros autores (15) (16) determinan los parámetros ingenieriles de suelos expansivos mediante la carta de plasticidad, la clasificación USC y la curva granulométrica.

En este estudio se determinaron, para cada muestra, los límites de Atterberg (Límite Líquido: LL, Límite Plástico: LP, e índice Plástico: IP) representando las muestras en la carta de plasticidad; se llevó a cabo un estudio granulométrico (incluyendo el gráfico de la granulometría del suelo); y se clasificaron las muestras según la USCS y la AASHTO.

Los ensayos de laboratorio se realizaron conforme a lo establecido en las normas UNE: preparación previa de la muestra (17); humedad por secado de estufa (18); granulometría de suelos finos por sedimentación (19), Límite Líquido (20); Límite Plástico (21) y granulometría por tamizado (22) (23) (24) (25).

\subsection{Incidencia que ejerce el tipo de suelo en las condiciones ambientales en el interior de las bodegas}

La temperatura en el interior de una construcción subterránea está fuertemente condicionada por la temperatura del terreno a la profundidad de excavación (7). La transferencia de calor en el suelo depende de las características del terreno. El amortiguamiento y desfase de la temperatura del exterior están directamente relacionados con la conductividad térmica $(\kappa)$, calor específico $(c)$ y densidad $(\rho)$ del terreno, pudiendo agruparse estos tres parámetros en la difusividad térmica $(\alpha=\kappa / \rho c)$ del mismo.

El amortiguamiento o reducción de la amplitud térmica del exterior es más pronunciado en suelos ligeros (baja $\alpha$ ), mientras que en suelos pesados (alta $\alpha$ ), las fluctuaciones son conducidas a mayor profundidad (26). La influencia de la difusividad es suficientemente grande para que el rango anual de fluctuación de la temperatura a ciertas profundidades pueda variar más del doble dependiendo de las características del terreno (26). Variando la profundidad en función del tipo de terreno y las condiciones del exterior podemos conseguir (en el interior de las bodegas subterráneas) un factor de amortiguamiento deseado y una reducción considerable de la temperatura máxima anual en cualquier localización (27).

Asumiendo la simplificación de que el terreno es un sólido homogéneo semi-infinito con conducción de calor unidimensional y propiedades físicas constantes y que la temperatura de la superficie del suelo varía periódicamente con el tiempo, la reducción del intervalo de temperatura en función de la profundidad se puede estimar a través de (26) :

$$
\emptyset=\frac{A_{x}}{A_{S}}=e^{-x \sqrt{\frac{\pi}{365 \alpha}}}
$$

Donde:

$\varnothing=$ Factor de decremento

$x=$ Profundidad (m).

$A_{x}=$ Amplitud de la onda térmica a la profundidad $x\left({ }^{\circ} \mathrm{C}\right)$.

$A_{s}=$ Amplitud de la onda térmica en la superficie $\left({ }^{\circ} \mathrm{C}\right)$.

$\alpha=$ Difusividad térmica aparente $\left(\mathrm{m}^{2} / \mathrm{s}\right)$.

\section{RESULTADOS}

\subsection{Diferencias entre las muestras de una misma localización}

Tras el análisis en el laboratorio se caracterizaron y clasificaron todas las muestras, comparando el resultado de las muestras interiores frente a las exteriores (Tabla 1).

A la vista de los parámetros y de la clasificación de las muestras se concluye que para el objetivo del estudio las diferencias en 5 de los 6 casos son pequeñas. Las diferencias de clasificación entre muestras interiores y exteriores se deben a que los valores están muy próximos a los límites de separación entre categorías, presentando en realidad propiedades muy similares. Las diferencias de granulometría en Torragalindo apenas afectan a la clasificación y a los límites de plasticidad.

La excepción es el caso de San Juan del Monte, donde existe una diferencia apreciable en la plasticidad, pudiendo ser debida a la adicción posterior de tierra de distinta procedencia sobre el cañón.

A la vista de los resultados, y teniendo en cuenta el elevado número de muestreos a realizar $(30 \%$ del total de barrios de bodegas existentes) y el objeto del estudio, hemos asumido la simplificación de que el exterior del cañón presenta un suelo de 
características similares al interior, que en su día se extrajo de la cueva y se depositó sobre el cañón. De esta forma, en aquellos barrios de bodegas en que no fue posible tomar muestras interiores se obtuvieron del exterior del cañón.

\subsection{Estudio de los suelos}

Los resultados del análisis en el laboratorio y clasificación de las muestras de suelos tomadas en cada uno de los 31 barrios de bodegas se muestran en la Tabla 2.

Tabla 1. Comparación de las muestras de suelo interiores frente a las exteriores

\begin{tabular}{|c|c|c|c|c|c|c|c|c|c|}
\hline \multirow[b]{2}{*}{ Localización } & \multirow[b]{2}{*}{ Tipo } & \multicolumn{4}{|c|}{ Granulometría (\% que pasa) } & \multicolumn{2}{|c|}{ Límites de Atterberg } & \multicolumn{2}{|c|}{ Clasificación } \\
\hline & & $\mathrm{T}-4$ & $\mathrm{~T}-10$ & $\mathrm{~T}-40$ & T-200 & $\begin{array}{c}\text { L.L. } \\
\text { (Límite Líquido) }\end{array}$ & $\begin{array}{c}\text { I.P. } \\
\text { (Índice plástico) }\end{array}$ & AASHTO & USCS \\
\hline \multirow{2}{*}{ Atauta } & Exterior & 90 & 84 & 72 & 34 & 22 & 7 & A-2-4 & SM-SC \\
\hline & Interior & 90 & 86 & 78 & 49 & 23 & 7 & A-4 & SM-SC \\
\hline \multirow{2}{*}{ Hoyales de Roa } & Exterior & 99 & 99 & 96 & 44 & - & No plástico & A-4 & SM \\
\hline & Interior & 97 & 96 & 89 & 18 & $\begin{array}{llll}- & \\
\end{array}$ & No plástico & A-2-4 & SM \\
\hline \multirow{2}{*}{$\begin{array}{l}\text { Montejo de la Vega } \\
\text { de la Serrezuela }\end{array}$} & Exterior & 100 & 99 & 97 & 94 & 38 & 14 & A-6 & $\mathrm{CL}$ \\
\hline & Interior & 100 & 99 & 97 & 88 & 41 & 13 & A-7-6 & ML \\
\hline \multirow{2}{*}{ San Juan del Monte } & Exterior & 98 & 97 & 91 & 60 & 32 & 8 & A-4 & $\mathrm{ML}$ \\
\hline & Interior & 97 & 94 & 92 & 32 & - & No plástico & A-2-4 & SM \\
\hline \multirow{2}{*}{ Torregalindo } & Exterior & 62 & 57 & 54 & 53 & 56 & 26 & A-7-5 & $\mathrm{MH}$ \\
\hline & Interior & 97 & 85 & 77 & 73 & 51 & 22 & A-7-6 & $\mathrm{MH}$ \\
\hline \multirow{4}{*}{$\begin{array}{l}\text { San Esteban } \\
\text { de Gormaz }\end{array}$} & Exterior & 90 & 77 & 63 & 56 & 27 & 8 & A-4 & $\mathrm{CL}$ \\
\hline & Exterior & 97 & 89 & 79 & 69 & 26 & 9 & A-4 & $\mathrm{CL}$ \\
\hline & Exterior & 91 & 83 & 76 & 69 & 27 & 10 & A-4 & $\mathrm{CL}$ \\
\hline & Interior & 100 & 97 & 86 & 79 & 27 & 9 & A-4 & $\mathrm{CL}$ \\
\hline
\end{tabular}

Tabla 2. Propiedades de los 31 suelos muestreados en que están excavadas las bodegas subterráneas

\begin{tabular}{|c|c|c|c|c|c|c|c|c|c|c|}
\hline \multirow{2}{*}{ Código } & \multirow{2}{*}{ Localización } & \multirow{2}{*}{ Tipo } & \multicolumn{4}{|c|}{ Granulometría (\% que pasa) } & \multicolumn{2}{|c|}{ Límites de Atterberg } & \multicolumn{2}{|c|}{ Clasificación } \\
\hline & & & $\mathrm{T}-4$ & $\mathrm{~T}-10$ & $\mathrm{~T}-40$ & $\mathrm{~T}-200$ & $\begin{array}{c}\text { L.L. } \\
\text { (Límite Líquido) }\end{array}$ & $\begin{array}{c}\text { I.P. } \\
\text { (Índice plástico) }\end{array}$ & AASHTO & USCS \\
\hline 1 & Aldeyuso & Interior & 96 & 96 & 92 & 65 & 39 & 14 & A-6 & $\mathrm{CL}$ \\
\hline 2 & Atauta & Interior & 90 & 86 & 78 & 49 & 23 & 7 & A-4 & SM-SC \\
\hline 3 & Hoyales de Roa & Interior & 97 & 96 & 89 & 18 & - & No plástico & A-2-4 & SM \\
\hline 4 & Langa de Duero & Interior & 99 & 95 & 91 & 88 & 44 & 21 & A-7-6 & $\mathrm{CL}$ \\
\hline 5 & Miño de San Esteban & Interior & 86 & 82 & 79 & 71 & 66 & 39 & A-7-6 & $\mathrm{CH}$ \\
\hline 6 & Montejo de la Vega de la S. & Interior & 100 & 99 & 97 & 88 & 41 & 13 & A-7-6 & $\mathrm{ML}$ \\
\hline 7 & Peñalba de San Esteban & Interior & 100 & 80 & 67 & 52 & 25 & 8 & A-4 & $\mathrm{CL}$ \\
\hline 8 & IRejas de San Esteban & Interior & 92 & 92 & 91 & 47 & - & No plástico & A-4 & SM \\
\hline 9 & San Esteban de Gormaz & Interior & 100 & 97 & 86 & 79 & 27 & 9 & A-4 & $\mathrm{CL}$ \\
\hline 10 & San Juan del Monte & Interior & 97 & 94 & 92 & 32 & - & No plástico & A-2-4 & SM \\
\hline 11 & Soto de San Esteban & Interior & 99 & 99 & 95 & 42 & - & No plástico & A-4 & SM \\
\hline 12 & Torregalindo & Interior & 97 & 85 & 77 & 73 & 51 & 22 & A-7-6 & $\mathrm{MH}$ \\
\hline 13 & Velilla de San Esteban & Exterior & 85 & 77 & 69 & 62 & 32 & 10 & A-4 & $\mathrm{CL}$ \\
\hline 14 & Alcubilla del Marqués & Exterior & 96 & 93 & 86 & 60 & 20 & 7 & A-4 & $\mathrm{ML}-\mathrm{CL}$ \\
\hline 15 & Boada de Roa & Exterior & 98 & 95 & 90 & 43 & - & No plástico & A-4 & SM \\
\hline 16 & Castillejo de Robledo & Exterior & 58 & 50 & 44 & 39 & 40 & 11 & A-6 & SC \\
\hline 17 & Castrillo de Duero & Exterior & 100 & 100 & 96 & 64 & 35 & 11 & A-6 & $\mathrm{CL}$ \\
\hline 18 & Castrillo de la Vega & Exterior & 99 & 96 & 94 & 82 & 35 & 11 & A-6 & $\mathrm{CL}$ \\
\hline 19 & Fuentecén & Exterior & 96 & 94 & 83 & 30 & - & No plástico & A-2-4 & SM \\
\hline 20 & Fuentelcésped & Exterior & 99 & 95 & 89 & 64 & 59 & 26 & A-7-5 & $\mathrm{MH}$ \\
\hline 21 & Fuentelisendo & Exterior & 96 & 93 & 89 & 42 & 27 & 9 & A-4 & SC \\
\hline 22 & Matanza de Soria & Exterior & 88 & 82 & 77 & 53 & 23 & 5 & A-4 & $\mathrm{ML}-\mathrm{CL}$ \\
\hline 23 & Nava de Roa & Exterior & 94 & 93 & 88 & 43 & 33 & 13 & A-6 & SC \\
\hline 24 & Olmedillo de Roa & Exterior & 94 & 90 & 74 & 34 & 21 & 5 & A-2-4 & SM-SC \\
\hline 25 & Quintana del Pidio & Exterior & 99 & 98 & 95 & 60 & 27 & 8 & A-4 & $\mathrm{CL}$ \\
\hline 26 & Quintanilla de Arriba & Exterior & 99 & 97 & 90 & 75 & 50 & 17 & A-7-5 & $\mathrm{MH}$ \\
\hline 27 & San Martín de Rubiales & Exterior & 100 & 100 & 97 & 30 & - & No plástico & A-2-4 & SM \\
\hline 28 & Sinovas & Exterior & 100 & 99 & 91 & 31 & - & No plástico & A-2-4 & SM \\
\hline 29 & Valbuena de Duero & Exterior & 93 & 91 & 83 & 63 & 47 & 10 & A-5 & $\mathrm{ML}$ \\
\hline 30 & Valdearcos de la Vega & Exterior & 99 & 99 & 96 & 66 & 36 & 12 & A-6 & $\mathrm{CL}$ \\
\hline 31 & Villálvaro & Exterior & 96 & 92 & 86 & 69 & 28 & 11 & A-6 & $\mathrm{CL}$ \\
\hline
\end{tabular}




\subsubsection{Clasificación}

\section{- Unified Soil Classification System (USCS)}

Según los criterios de esta clasificación, predominan los suelos limo-arcillosos de baja plasticidad $(\mathrm{CL}, \mathrm{ML}$ y $\mathrm{ML}-\mathrm{CL})$ con un $45 \%$ de las muestras, seguido de suelos arenosos con alto porcentaje de partículas finas y plasticidad baja o nula (SM, SC y SM-SC) con un $42 \%$ (Figura 7 ).

\section{USCS}

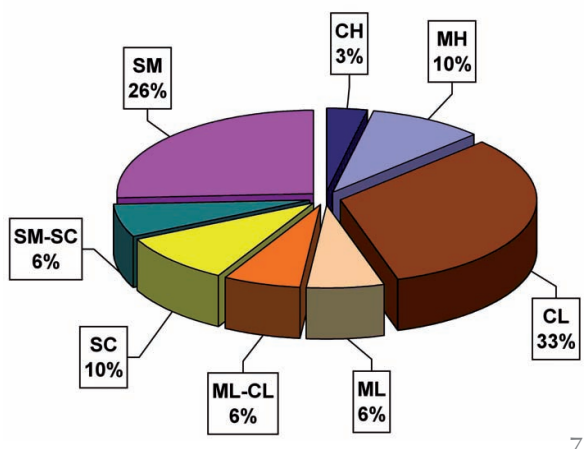

En las 31 localizaciones muestreadas no se han encontrado bodegas excavadas en terrenos con alto contenido de gravas (GW, GP, GM, GC) ni de arenas limpias (SW, SP) que se hayan conservado hasta nuestros días. Por otra parte, las bodegas excavadas en terrenos limo arcillosos de alta plasticidad (LL>50) son escasos (13\%).

\section{- AASHTO}

Una de las principales diferencias entre esta clasificación y la USCS es que fija el límite entre los suelos de partículas finas (arcillosos y limosos) y los suelos de partículas gruesas (gravas y arenas) en un porcentaje de paso por tamiz 200 del $36 \%$, en lugar del $50 \%$ que fijaba la USCS. El resultado es que un gran número de muestras clasificadas como suelos arenosos con alto porcentaje de partículas finas son ahora clasificadas como suelos limosos o arcillosos. Además, la diferencia entre alta y baja plasticidad se reduce de L.L. 50 a 40, con lo que varias muestras con L.L. entre 40 y 50 pasan a considerarse como alta plasticidad.

De esta forma, el resultado de la clasificación se resume en la Figura 8. Los suelos más habituales en la construcción de bodegas son los suelos limosos de baja o nula plasticidad (A-4), seguidos por suelos arciIlosos (A-6) y gravas y arenas limo-arcillosas (A-2-4), también de baja plasticidad. Los suelos de alta plasticidad representan más de un $20 \%$ de las muestras. La presencia de otros suelos es prácticamente despreciable. Esta clasificación presenta la desventaja de agrupar en una misma categoría (A-4) muestras arenosas con plasticidad nula y muestras con mayor porcentaje de partículas finas e IP próximo a 10. Por el contrario, la clasificación USCS agrupa todas las muestras con plasticidad nula en una misma categoría (SM).

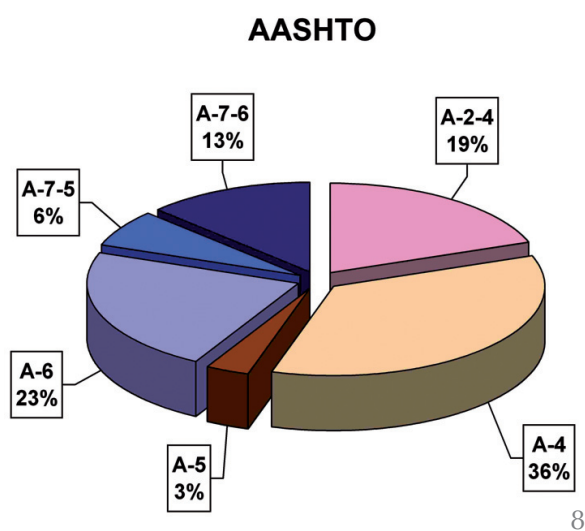

- Resultados

Según los resultados de las clasificaciones, se puede concluir que los suelos predominantes en las bodegas que han resistido hasta nuestros días son suelos con alto porcentaje de partículas finas (limosos, arcillosos y arenas limosas o arcillosas) de baja o nula plasticidad. La presencia de suelos con alta plasticidad es reducida. No se han encontrado suelos con alto contenido en partículas gruesas.

En la Figura 9 se puede ver la relación entre la distribución de las muestras en la zona de estudio y su clasificación. Se puede observar que existe cierta zonificación de las diferentes categorías a lo largo de la región, presentando amplias zonas con características similares. Así por ejemplo, utilizando la clasificación AASHTO, se observa como en la zona soriana, predominan suelos limosos (A-4) con menor proporción de arcillosos (A-6 y A-7); en la provincia de Valladolid los suelos arciIlosos (A-6 y A-7); mientras que en la provincia de Burgos aumenta la proporción de suelos más granulares (A-2). Es destacable que las muestras exteriores e interiores próximas presentan igual o similar clasificación.

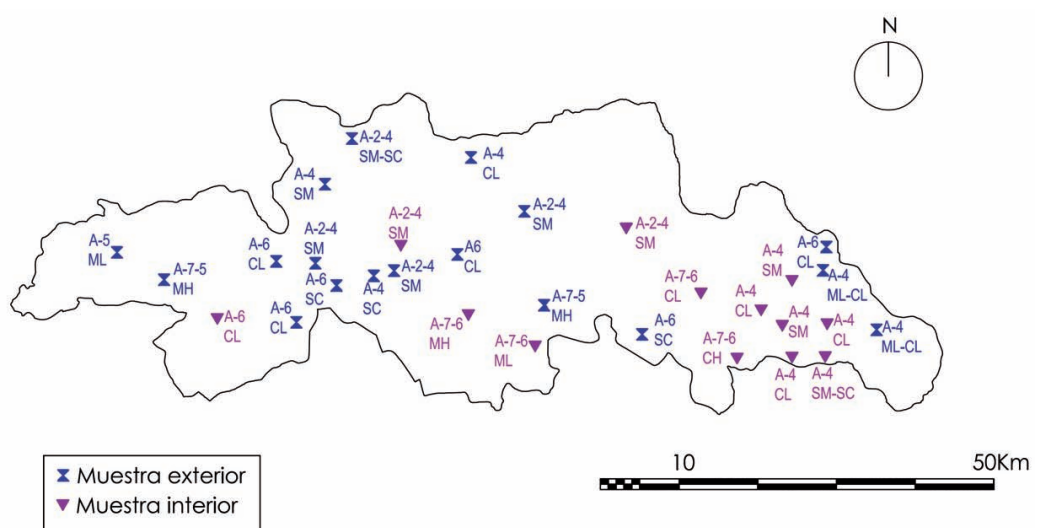

7. Clasificación USCS de las 31 muestras de suelo en que están excavadas las bodegas subterráneas

8. Clasificación AASHTO de las 31 muestras de suelo en que están excavadas las bodegas subterráneas.

9. Clasificación USCS y AASHTO de las muestras según localización. 
10. Carta de plasticidad de las muestras con plasticidad no nula.

11. Rango de distribución de tamaños de las partículas de los suelos en que están excavadas las bodegas.
No obstante, las diferencias entre las dos clasificaciones ponen de manifiesto que muestras de características muy similares son clasificadas en diferentes grupos en función de los criterios de la clasificación seleccionada, pudiendo inducir a error si se consideran las propiedades generales del grupo. Parece necesario complementar la información de las clasificaciones con un estudio detallado de plasticidad y con un estudio granulométrico.

\subsubsection{Plasticidad}

Según diferentes autores (14) (15) (16) la plasticidad está directamente relacionada con la expansibilidad del suelo. Por ello, para estudiar las características y similitudes de las muestras más allá de las clasificaciones se han representado en la carta de plasticidad las muestras con plasticidad no nula. Se entiende por baja plasticidad a la zona con $\mathrm{LL}<35$; media $35<\mathrm{LL}<50$; alta $50<\mathrm{LL}<70$ y muy alta $\mathrm{LL}>70$.

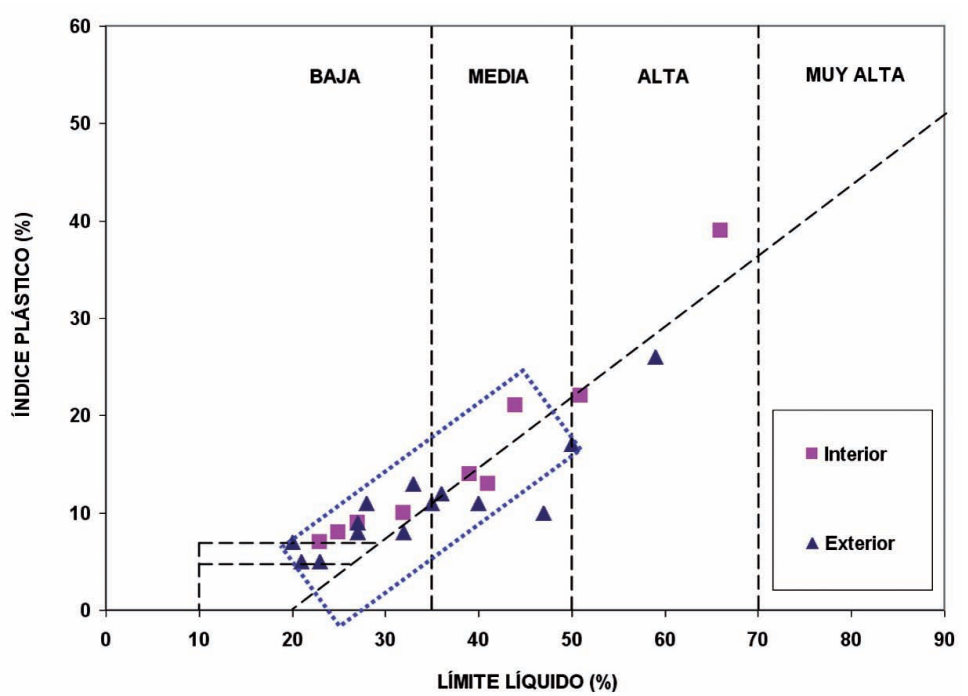

10

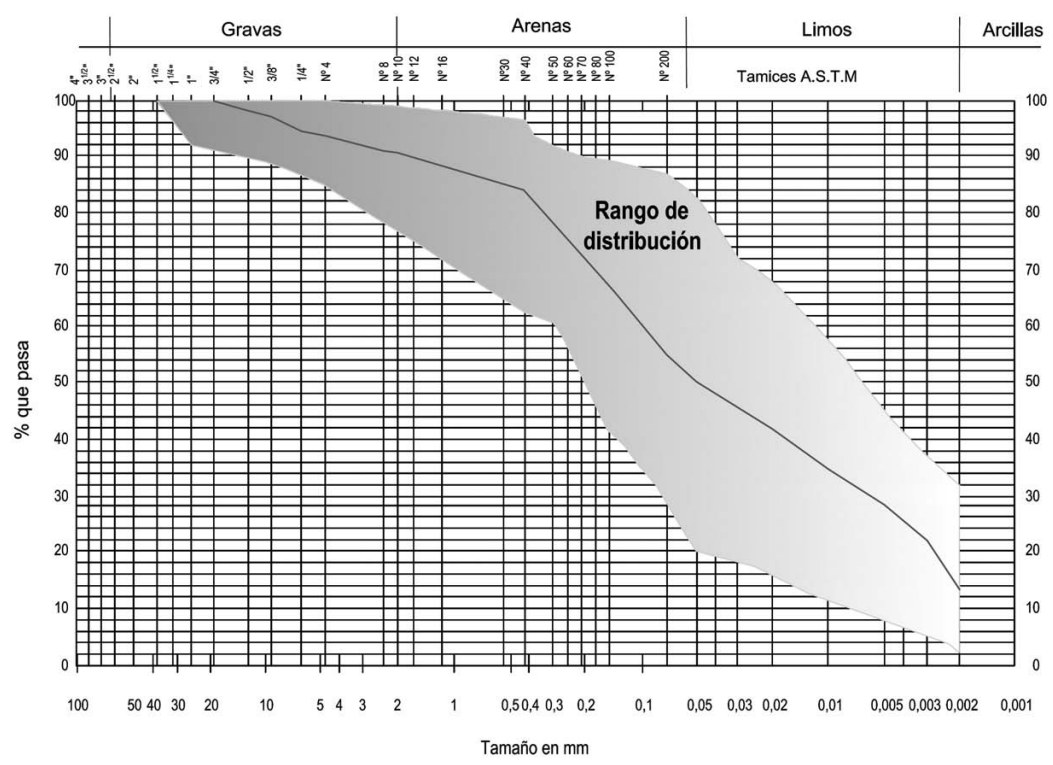

En la Figura 10 se observa como la mayoría de las muestras, con independencia de la granulometría, presentan unas características muy similares, agrupadas en la zona de baja y media plasticidad (LL entre 20 y 50; IP entre 5 y 15), próximas a las líneas que dividen los limos y arcillas en ambas clasificaciones. Esto explica la variabilidad de clasificación en categorías próximas.

Tan sólo dos muestras parecen presentar riesgos de sufrir cambios de volumen y afectar a la integridad de la construcción dada su alta plasticidad. No se han encontrado suelos con muy alta plasticidad.

Por lo tanto, excluyendo las muestras de plasticidad nula (26\%), en la zona que delimitan la mayoría de las muestras predominan los terrenos de baja o media plasticidad.

\subsubsection{Granulometría}

La granulometría de la zona de estudio se muestra en la Figura 11, con el rango de distribución de tamaños de las partículas de los suelos y su valor promedio para el total de muestras. Se ha excluido de la representación la curva de la muestra con código de localización 16, al mostrar una tendencia muy diferente al resto.

Según la norma UNE ISO 14688-1:2002 (28), se denominan arcillas a las partículas con tamaño $\leq 0,002 \mathrm{~mm}$; limos de 0,002 a $0,063 \mathrm{~mm}$; arenas de 0,063 a 2,0 $\mathrm{mm}$ y gravas de $2,0 \mathrm{~mm}$ a $63 \mathrm{~mm}$. El promedio de las muestras estudiadas contiene una proporción de arcillas del $14 \% \pm 10$; limos $38 \% \pm 17$; arenas $38 \% \pm 19$ y gravas $10 \% \pm 10$. Del porcentaje de arenas, un $32 \%$ tienen un tamaño de partícula menor de $0,4 \mathrm{~mm}$ y el $6 \%$ restante mayor de $0,4 \mathrm{~mm}$.

La gran anchura del rango de distribución próxima a los 0,06 $\mathrm{mm}$ y el estrechamiento en los extremos refleja que la mayor variabilidad se produce en el límite entre arenas y limos, existiendo muestras con alto porcentaje de limos y muestras con altos porcentajes de arenas. Conforme nos alejamos de este punto las diferencias entre las muestras se van reduciendo, siendo el contenido en arcillas y gravas más uniforme.

El cambio de pendiente próximo al tamiz $40(0,4 \mathrm{~mm})$ de la curva promedio y su escasa variación hasta la separación de las arcillas refleja que desde el punto de vista granulométrico predominan los limos y arenas finas (en torno al $70 \%$ del total). 


\subsubsection{Incidencia del tipo de suelo en el ambiente interior}

Aplicando la metodología descrita se pueden calcular valores de reducción del intervalo anual de temperaturas a distintas profundidades para los suelos donde están excavadas las bodegas. Así por ejemplo, considerando un valor de difusividad térmica de $9,76 \times 10-7 \mathrm{~m}^{2} / \mathrm{s}$ para un suelo limo-arenoso (29), similar a los encontrados en muchas de las bodegas analizadas, el factor de decremento sería de 0,53 a $2 \mathrm{~m}, 0,28$ a $4 \mathrm{~m}, 0,15$ a $6 \mathrm{~m}, 0,08$ a $8 \mathrm{~m}, 0,04$ a $10 \mathrm{~m}$, etc.

En un terreno típico para la construcción de bodegas, la reducción del rango exterior de temperatura sería del $96 \%$ a 10 m de profundidad, con escasas variaciones a lo largo del año, transmitiendo esta estabilidad al ambiente interior.

\section{CONCLUSIONES}

El principal objetivo de esta investigación es determinar el terreno en que se sitúan las bodegas subterráneas centenarias existentes en una zona con gran tradición vitivinícola y elevado número de bodegas subterráneas: La Ribera del Duero (España).

Se han identificado y clasificado todos los barrios de bodegas existentes en la región que han perdurado hasta nuestros días, contabilizando 106. Se han seleccionado y analizado muestras de suelo en 31 barrios de bodegas diseminados por toda la región. Las muestras se han clasificado según la USCS y la AASHTO, Ilevando a cabo además un estudio individualizado de granulometría y de plasticidad.

A la hora de determinar la idoneidad de un terreno para excavar una bodega subterránea es necesario combinar la información obtenida de las clasificaciones USCS y/o AASHTO y el estudio individualizado de la plasticidad y la granulometría. El empleo de las dos clasificaciones ayuda a caracterizar los suelos.

Los suelos más habituales son los CL y SM según la USCS y los A-4, A-6 y A-2-4 según la AASHTO.

Los resultados demuestran que la mayoría de los suelos en que están excavadas las bodegas presentan unas características de plasticidad y expansibilidad muy similares.
Se concentran en una región muy concreta de la carta de plasticidad $(20<\mathrm{LL}<50$ $5<\mathrm{IP}<15$ ) o presentan plasticidad nula. Los suelos con elevada plasticidad $(50<\mathrm{LL}<70)$ no son habituales (en torno al $10 \%$, pudiendo ser debido al mayor riesgo de sufrir expansiones y provocar desprendimientos y el colapso de la estructura. No se han encontrado bodegas excavadas en terrenos con muy alta plasticidad (LL > 70\%).

Podemos afirmar que los suelos donde se ubican las bodegas en la zona de estudio están formados principalmente por arenas y limos. No se han encontrado bodegas excavadas en terrenos donde predominen gravas o arenas limpias.

Las propiedades del terreno van a condicionar la transferencia de calor, consiguiendo mayor estabilidad térmica a una profundidad dada cuanto menor sea la difusividad térmica del suelo.

La caracterización de los suelos en los que han sido construidas las bodegas de estudio, a pesar de limitarse a una zona concreta, sirve de referencia para la búsqueda de suelos similares en otras localizaciones, que aseguren la durabilidad e integridad de la construcción, ya que están basados en bodegas que se han conservado durante decenas o incluso centenares de años. Esto no implica la no existencia de otros tipos de suelos también validos para la construcción de bodegas subterráneas.

Así, los resultados de este trabajo podrían ser usados para el diseño de nuevas construcciones con similares características, a imagen de las bodegas subterráneas tradicionales centenarias que han soportado sin problemas el paso del tiempo, proporcionando durante siglos unas condiciones óptimas para la crianza del vino.

\section{AGRADECIMIENTOS}

Este estudio ha sido realizado dentro del proyecto BIA2004-03266, "La construcción subterránea para bodegas. Sistemas de eco-construcción para vinos de calidad", financiado por el Ministerio de Educación de España.

Agradecemos al ingeniero agrónomo Sergio Díaz Pérez su colaboración en esta investigación.

\section{BIBLIOGRAFÍA}

(1) Bestraten, S.; Hormías, E.; Altemir, A.: "Construcción con tierra en el siglo XXI", Informes de la Construcción, vol. 63 (523) 2011, pp. 5-20. 
(2) Cid, J.; Mazarrón, F. R.; Cañas, I.: "Las normativas de construcción con tierra en el mundo", Informes de la Construcción, vol. 63, nº 523 (2011), pp. 159-169.

(3) Mazarrón, F. R.; Cañas, I.: "Seasonal analysis of the thermal behaviour of traditional underground wine cellars in spain", Renewable Energy, vol. 34, n 11, 2009, pp. 2484-2492.

(4) Silvia, M. O.; Ignacio, C. G.: "Comparison of hygro-thermal conditions in underground wine ceIlars from a spanish area", Building and Environment, vol. 40, n 10, 2005, pp. 1384-1394.

(5) Martín Ocaña, S.; Cañas Guerrero, I.: "Comparison of analytical and on site temperature results on spanish traditional wine cellars", Applied Thermal Engineering, vol. 26, n 7, 2006, pp. 700-708.

(6) Martin, S.; Canas, I.: "A comparison between underground wine cellars and aboveground storage for the aging of spanish wines", Transactions of the Asabe, vol. 49, n 5, 2006, pp. 1471-1478.

(7) Mazarrón, F. R.; Cañas, I.: "Exponential sinusoidal model for predicting temperature inside underground wine cellars from a spanish region", Energy and Buildings, vol. 40, n 10, 2008, pp. 1931-1940.

(8) Farrokh, E.; Mortazavi, A.; Shamsi, G.: "Evaluation of ground convergence and squeezing potential in the TBM driven ghomroud tunnel project", Tunnelling and Underground Space Technology, vol. 21, no 5, 2006, pp. 504-510.

(9) Goel, R. K.; Swarup, A.: "A case history of tunnelling through difficult ground", Tunnelling and Underground Space Technology, vol. 21 n 3-4, 2006, pp. 362-362.

(10) Grandori, R.: "Abdalajis east railway tunnel (spain) - double shield universal TBM cope with extremely poor and squeezing formations", Tunnelling and Underground Space Technology, vol. 21 n³-4, 2006, pp. 268-268.

(11) Pérez-Romero, J.; Oteo, C. S.; De la Fuente, P.: "Design and optimisation of the lining of a tunnel in the presence of expansive clay levels", Tunnelling and Underground Space Technology, vol. 22 $\mathrm{n}^{\mathrm{o}}$ 1, 2007, pp. 10-22.

(12) Pardo, J. M. F.; Guerrero, I. C.: "Subterranean wine cellars of central-Spain (Ribera de Duero): An underground built heritage to preserve", Tunnelling and Underground Space Technology, vol. 21, $\mathrm{n}^{\circ}$ 5, 2006, pp. 475-484.

(13) Doudkinski, D.; Frid, V.; Liskevich, G.; Prihodko, L.; Zlotnikov, R.: "Towards the digital indexation of USCS classification: Case study in Israel", Engineering Geology, vol. 95 n 1-2 (2007), pp. 48-55.

(14) Al-rawas, A. A.; Qamaruddin, M.: "Construction problems of engineering structures founded on expansive soils and rocks in northern oman", Building and Environment, vol. 33, n 2-3 (1998), pp. 159-171.

(15) Yitagesu, F. A.; Van der Meer, F.; Van der Werff, H.; Zigterman, W.: "Quantifying engineering parameters of expansive soils from their reflectance spectra", Engineering Geology, vol. 105, n 3-4 (2009), pp. 151-160.

(16) Abiddin Erguler, Z.; Ulusay, R.: "A simple test and predictive models for assessing swell potential of ankara (turkey) clay", Engineering Geology, vol. 67, no 3-4 (2003), pp. 331-352.

(17) UNE 103100:1995: Preparación de muestras para ensayos de suelos.

(18) UNE 103300:1993: Determinación de la humedad de un suelo mediante secado en estufa.

(19) UNE 103102:1995: Análisis granulométrico de suelos finos por sedimentación. Método del densímetro.

(20) UNE 103103:1994: Determinación del límite líquido de un suelo por el método del aparato de Casagrande.

(21) UNE 103104:1993: Determinación del límite plástico de un suelo.

(22) UNE-EN 933-1:1998: Ensayos para determinar las propiedades geométricas de los áridos. Parte 1: Determinación de la granulometría de las partículas. Métodos del tamizado.

(23) UNE-EN 933-1:1998/A1:2006: Ensayos para determinar las propiedades geométricas de los áridos. Parte 1: Determinación de la granulometría de las partículas. Métodos del tamizado.

(24) UNE-EN 933-2:1996: Ensayos para determinar las propiedades geométricas de los áridos. Parte 2: Determinación de la granulometría de las partículas. Tamices de ensayo, tamaño nominal de las aberturas.

(25) UNE-EN 933-2/1M:1999: Ensayos para determinar las propiedades geométricas de los áridos. Parte 2: Determinación de la granulometría de las partículas. Tamices de ensayo, tamaño nominal de las aberturas.

(26) Labs, K.: "Regional-Analysis of Ground and Above-Ground Climate Conclusion", Underground Space, 7, 1982, pp. 37-65.

(27) Mazarrón, F. R.; Cid-Falceto, J.; Cañas, I.: "An assessment of using ground thermal inertia as passive thermal technique in the wine industry around the world", Applied Thermal Engineering, 2012, pp. 33-34, 54-61.

(28) UNE ISO 14688-1:2002: Ingeniería geotécnica. Identificación y clasificación de suelos. Parte 1: Identificación y descripción.

(29) Reddy, K. R.; Cameselle, C.: Electrochemical Remediation Technologies for Polluted Soils, Sediments and Groundwater, John Wiley \& Sons, Inc., Hoboken, New Jersey, 2009. 